Available online at GSC Online Press Directory

GSC Biological and Pharmaceutical Sciences

e-ISSN: 2581-3250, CODEN (USA): GBPSC2

Journal homepage: https://www.gsconlinepress.com/journals/gscbps

(RESEARCH ARTICLE)

\title{
Audit of radiology reports of patients with neoplasms performed on computed tomography
}

\author{
Staver Natalia * and Rotaru Natalia \\ Department of radiology and medical imaging of "Nicolae Testemiţanu" State University of Medicine and Pharmacy, \\ Chişinău, Republic of Moldova.
}

Publication history: Received on 06 August 2020; revised on 14 August 2020; accepted on 15 August 2020

Article DOI: https://doi.org/10.30574/gscbps.2020.12.2.0254

\begin{abstract}
Evaluation of the peculiarities of radiology reports of patients with neoplasms of the abdomen and pelvis to optimize clinical imaging management. The study included 104 patients, divided into two groups, experimental group (L2) and control group (L1), repeatedly investigated by computed tomography (CT) in 2009-2019, a total of 440 examinations, of which 120 prospective examinations and 320 retrospective examinations, aged between 25 and 85 years, with primary tumors of the abdomen and pelvis. Four specific descriptive criteria were analyzed from radiology reports and we obtained the following results: specification of the scanning protocols in radiology reports ( $0 \%$ for L1 and $95.3 \%$ for L2), specifying the reason for the examination in radiology reports (100\% for L1 and 26.0\% for L2), use of international terminology in radiology reports $(0 \%$ for $\mathrm{L} 1$ and $74 \%$ for L2), patient follow-up in radiology reports (88.5\% for L1 and $59.8 \%$ for L2). Following the SWOT analysis of the radiology reports, we have developed a standardized model for describing CT images of patients with oncological pathologies of the abdomen and pelvis. The decision-making process was built on Strengths, eliminating Weaknesses, exploiting Opportunities and removing Threats.
\end{abstract}

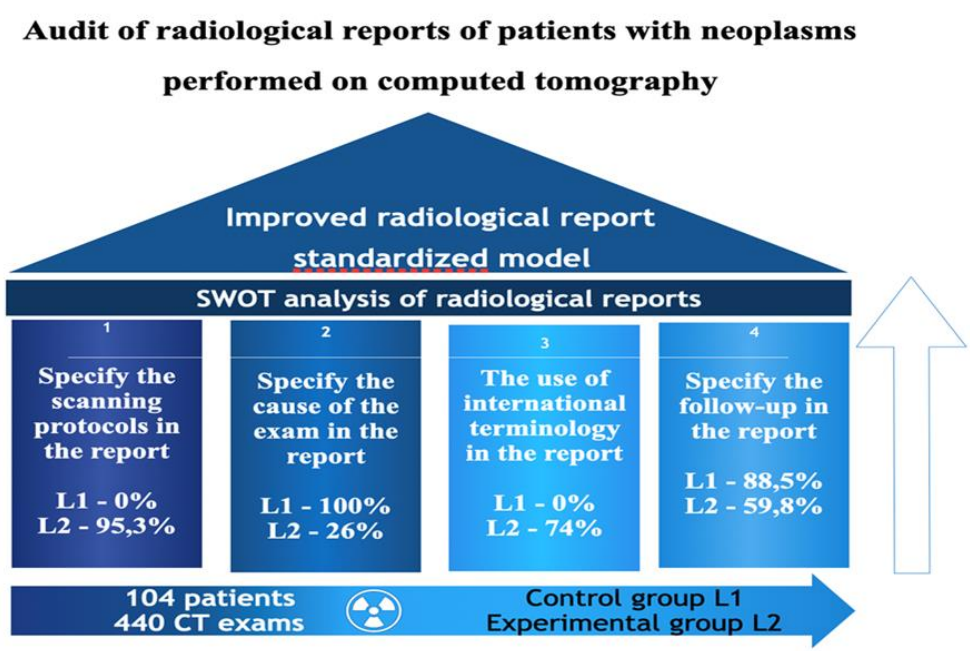

We evaluated 440 of CT reports of patients with abdominal and pelvic neoplasms reviewing four specific descriptive criteria that allowed us - by means of a SWOT analysis - to develop a standardized model for describing CT images of these patients.

Keywords: Computed tomography; Oncology; Radiology report; Imaging management; Follow-up.

\footnotetext{
${ }^{*}$ Corresponding author: Staver Natalia

Department of radiology and medical imaging of "Nicolae Testemițanu” State University of Medicine and Pharmacy, Chişinău, Republic of Moldova
} 


\section{Introduction}

Quality has become a predominant part of our lives. People are constantly looking for quality products and services. The existence of this desire for quality has led organizations around the world to consider it an essential component of any product and service. Quality is a tool of strategic differentiation to sustain competitive advantage. The quality of medical services is more difficult to define and measure than in other sectors. Distinctive features of the medical industry, such as intangibility, heterogeneity and simultaneity, make it difficult to define and measure quality. However, the quality of healthcare services depends on the service process and the interactions between doctors and patients [1, 2]. Some attributes of healthcare quality, such as timeliness, consistency, and accuracy are difficult to measure beyond a subjective assessment of the patient. Quality standards are more difficult to set in service operations. Healthcare professionals offer different services because the factors differ, such as experience, individual and personal skills [3].

Quality healthcare is a subjective, complex and multidimensional concept. Donabedian defined the quality of healthcare as the application of medical science and technology in a manner that maximizes its health benefits without adequately increasing the risk [4]. He distinguishes three components of quality: technical quality, interpersonal quality and facilities. Technical quality refers to the efficiency of health care. Interpersonal quality refers to the degree of satisfaction of the patient's needs and preferences. Facilities include environmental factors and the good organization of the provision of medical services [5]. Relating these notions to the topic of our study, the quality of the radiology report depends on: the quality of the medical equipment used to examine the patient, patient loyalty for cooperation and ensuring optimal management conditions for each patient in the Radiology Department.

\section{Material and methods}

To study the quality of the radiology report, patients with oncologic pathology of the abdomen and pelvis were examined. In order to achieve the purpose and objectives of the research, a controlled clinical diagnostic study was planned. The required number of patients for research was calculated using the following formula:

$n=\frac{1}{(1-f)} \times \frac{2\left(Z_{\alpha}+Z_{\beta}\right)^{2} \times P(1-P)}{\left(P_{o}-P_{1}\right)^{2}}$

where:

Po = According to the bibliographic data, the success of detecting the diagnosis of abdominal cancer by applying the traditional imaging algorithm is on average $63 \%$ (Po $=0.63)$;

P1 = In the research group the patients with abdominal cancer that will be investigated by the modified imaging algorithm, the success of the detection we assume will be in $90 \%$ of cases $(\mathrm{P} 1=0.90)$;

$\mathrm{P}=(\mathrm{P} 0+\mathrm{P} 1) / 2=0.77$

$\mathrm{Z} \alpha$ - tabular value. When the statistical significance is $95.0 \%$, then the coefficient $\mathrm{Z} \alpha=1.96$;

$\mathrm{Z} \beta$ - tabular value. When the statistical power of the comparison is $90.0 \%$, then the coefficient $\mathrm{Z} \beta=1.28$;

$\mathrm{f}=$ Proportion of subjects waiting to drop out of the study for reasons other than the investigated effect;

$\mathrm{q}=1 /(1-\mathrm{f}), \mathrm{f}=10.0 \%(0.1)$.

By entering the data in the formula we obtained 52 patients.

Criteria for inclusion in research:

- Adult patients (<18 years) with abdominal neoplasms who have signed the informed consent to participate in the study;

- Patients with abdominal neoplasms in stages I, II, III in the preoperative phase, postoperative phase and undergoing chemotherapy.

Exclusion criteria from the research:

- The patient's desire to leave the study;

- Uncooperative patients;

- Patients included in other ongoing clinical trials;

- Patients with abdominal neoplasms undergoing symptomatic treatment (stage IV).

In the absence of a standard scan or description of patients with oncological pathologies of the abdomen and pelvis, we considered it necessary to report the data of the experimental group (L2) to a control group (L1), with subsequent comparison of study results and useful practical recommendations of the descendants of the experimental group. Thus, two groups were created, 52 patients in each group, a total of 104 patients. The similarity of the groups being ensured in terms of age, sex, degree of disease activity, imaging parameters, the characteristics were evenly distributed; the 
batches were differentiated only from a geographical point of view, being considered alternatively, depending on the interest of the analysis, as experimental group (L2) and control group (L1).

The L1 research study included a number of 52 patients, repeatedly investigated by CT during 2009-2018, a total of 313 examinations, all of which were retrospective examinations. The average number of CT examinations recorded for a patient at the level of that group was 6.0 units $+\backslash-0.58$ units with a minimum value of 1.0 units and a maximum value of 20.0 units. The structure of the group according to sex was predominantly male (27 men, representing $51.9 \%$ ), women -25 , representing $48.1 \%$. The average age recorded in the group was $65.8+\backslash-1.32$ with a minimum of 39 years and a maximum of 85 years, with primary tumors of the esophagus, stomach, duodenum, pancreas, colon, rectum, cervical canal, ovaries, prostate, bladder, urethra.

The L2 research study included a number of 52 patients, repeatedly investigated by CT during 2013-2019, a total of 127 examinations, of which 120 prospective examinations and 7 retrospective examinations. The average number of CT examinations registered for a patient at the level of the respective group was 2.4 units $+\backslash-0.17$ units with a minimum value of 1.0 units and a maximum value of 7.0 units. The structure of the group according to sex was predominantly female (30 women, which represents $57.7 \%$ ), men - 22, which represents $42.3 \%$. The average age recorded in the group was $54.9+\backslash-1.53$ with a minimum of 25 years and a maximum of 77 years, with primary tumors of the stomach, liver, pancreas, adrenal glands, kidneys, bladder, rectum, uterus, ovaries, prostate, colon, rectum. The patients of the study were examined during the years 2009-2019, however it is known that patients must be followed for a long enough time to highlight the desired results.

The data collection source was the PACS (Picture Archiving and Communication Systems) and RIS (Radiological Information System) of two medical institutions, which meet the requirements of the study, in conditions of full confidentiality.

Statistical data processing was performed using the Microsoft Excel, Microsoft Office, IBM SPSS Statistics V22.0 software package, dedicated to epidemiological studies. The studies were carried out in accordance with the Helsinki Declaration of 1975, as revised in 2000 and with the approval of the ethics committee. All patients signed informed consent forms at the time of examination.

\section{Results}

In addition to professional experience, continuous medical development, interdepartmental communication, which directly contributes to the development of a qualitative radiology report, the radiologist examining a patient with an oncologic pathology must take into account specific descriptive criteria, such as:

- $\quad$ specifying the scanning protocols in the radiology report, in order to determine the quality of the radiology procedure;

- $\quad$ specifying the reason of the examination and anamnesis of the disease in the radiology report, which increases the degree of attention of the radiologist;

- $\quad$ the use of international terminology, especially as regards the interpretation of the response to therapy;

- comparison of current radiological images with previous radiological images (if the patient is not on initial assessment) - follow-up.

All these specific descriptive criteria were analyzed in the research groups and we have obtained the following results:

\subsection{Specification of scanning protocols in the radiology report}

In the radiology reports of the patients of the L1 research group, the specification of the scanning protocols was not indicated in $100 \%$ of the 313 examinations.

The specification of the scanning protocols in the radiology reports of the 52 patients from the L2 research group was performed in 121 examinations out of the 127 existing ones, which represents $95.3 \%$, but in 6 cases it was not indicated, which represents $4.7 \%$ from the total examinations of the $\mathrm{L} 2$ research group.

Comparing the frequency distributions using Pearson's X2 (chi square) test we obtain the value of X2 calculated $(411,328)$ at the degree of freedom $\mathrm{GL}=1$ and $p<0.001$. The calculated X2 value is much higher than the values found in table X2 for any of the three assumed risks (5\%,1\%, 0.1\%), so the difference between the two frequency distributions is statistically significant, denoting that within the sample there are different conditions that have determined this difference. 


\subsection{Specifying the reason of the examination in the radiology report}

The reason of the examination in the radiology reports of the 52 patients from the L1 research group was mentioned in $100 \%$ of the 313 examinations, including oncologic follow-up care - in 229 examinations among the existing ones, which represents $73.2 \%$, postsurgical restaging - in 37 examinations, which represents $0.6 \%$, pre-interventional staging - in 22 examinations, which represents $7.0 \%$, staging - in 19 examinations, which represents $0.6 \%$, restaging - in 4 examinations, which represents $1.3 \%$, initial assessment - in 2 examinations, which represents $0.6 \%$ of the total examinations of the L1 research group (Fig.1).

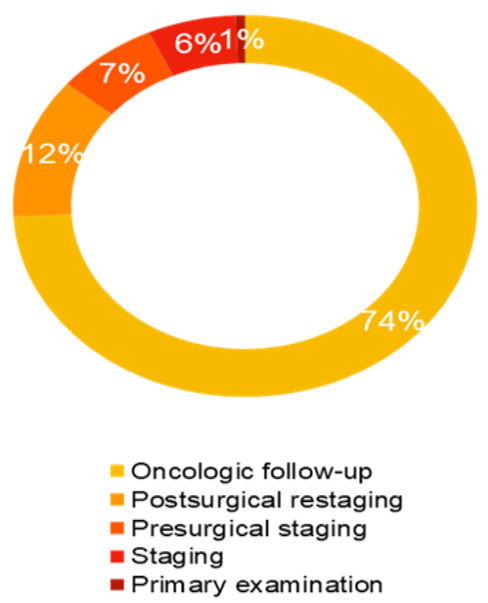

Figure 1 Specifying the cause of the exam. in L1

The reason of the examination in the radiology reports of the 52 patients in the L2 research group was mentioned in 33 examinations out of the existing 127 , which represents $26.0 \%$, but in 94 cases it was not indicated, which represents $74.0 \%$ of the total examinations of the L2 research group (Fig.2).

Comparing the frequency distributions using Pearson's X2 (chi square) test we obtain the calculated value of X2 $(440,000)$ at the degree of freedom GL $=7$ and $p<0.001$, suggesting a statistically significant link (HS, 99.9\% confidence).

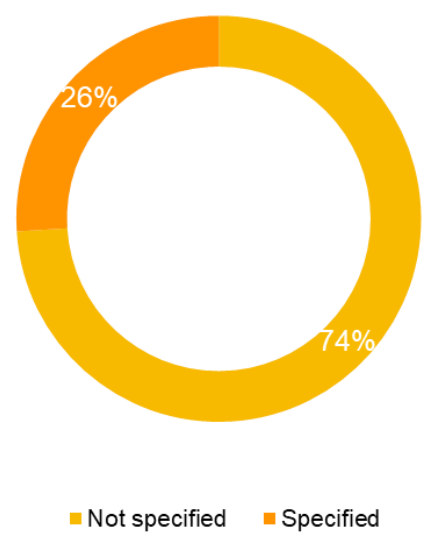

Figure 2 Specifying the cause of the exam. in L2

\subsection{Use of international terminology in the radiology report}

In the radiology reports of the patients of the $\mathrm{L} 1$ research group, the use of international terminology was not noticed in $100 \%$ of the 313 examinations (Fig.3).

The use of international terminology in the radiology reports of the 52 patients in the L2 research group was performed in 94 examinations out of the existing 127, which represents 74\%, but in 33 cases it was not indicated, which represents $26.0 \%$ of the total examinations L2 research group (Fig.4).

Comparing the frequency distributions using Pearson's X2 (chi square) test we obtain the calculated value of X2 $(294,608)$ at the degree of freedom GL $=1$ and $p<0.001$, suggesting a statistically significant link (HS, 99.9\% confidence). 


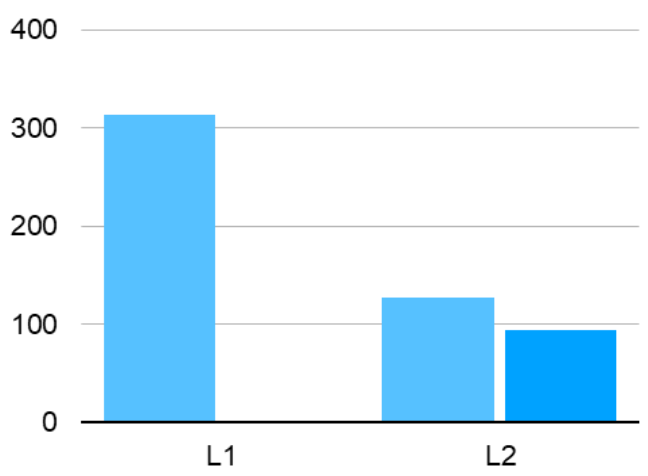

Figure 3 Use of IT in L1 radiology reports

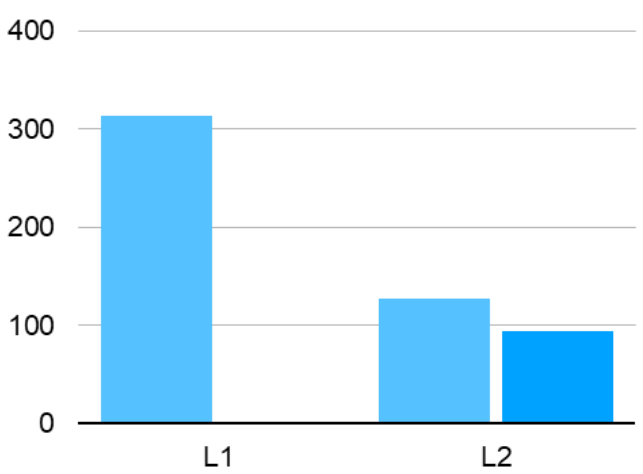

Figure 4 Use of IT in L2 radiology reports

\subsection{Specifying the follow-up in the radiology report}

In the L1 research group, 52 patients were examined, a total of 313 examinations, investigations in which the imaging data were compared with the previous examinations - 277 examinations, which represent $88.5 \%$, investigations in which the imaging data were not compared with the examinations previous - 36 examinations, which represent $11.5 \%$ of the total examinations of the L1 research group (Fig.5).

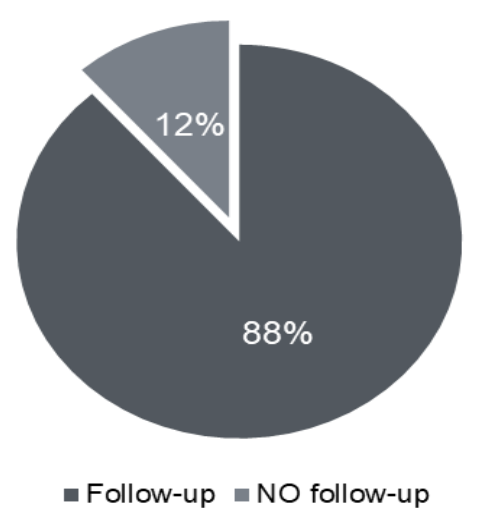

Figure 5 L1 follow-up

In the L2 research group, 52 patients were examined, a total of 127 examinations, of which 46 initial assessments, representing $36.2 \%$, investigations in which the imaging data were compared with previous examinations - 76 examinations, representing $59.8 \%$, investigations in which the imaging data were not compared with the previous examinations - 5 examinations, which represent $3.9 \%$ of the total examinations of the L2 research group (Fig.6).

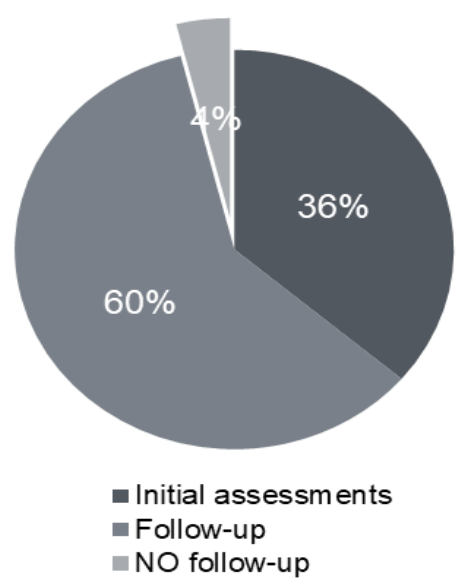

Figure 6 L2 follow-up 
Comparing the frequency distributions using Pearson's X2 (chi square) test we obtain the value of X2 calculated $(128,165)$ at the degree of freedom $\mathrm{GL}=2$ and $\mathrm{p}<0.001$. The calculated $\mathrm{X} 2$ value is much higher than the values found in table X2 for any of the three assumed risks (5\%,1\%, 0.1\%), so the difference between the two frequency distributions is statistically significant, denoting that within the sample there are different conditions that have determined this difference.

\section{Discussion}

Interpreting the specific oncology data from the analyzed radiology reports, we find a significant difference in the specification of scanning protocols in the radiology report and the use of international terminology in the radiology report, because in research group L1 the reported proportion of these two characteristics was $0 \%$ (Table 1 ), so it requires their urgent implementation in the description routine to meet the needs of clinicians who continue to manage these patients.

On the other hand, regarding the specification of the examination reason in the radiology report, there is a $100 \%$ rate in the L1 research group, compared to the merely $26 \%$ of the L2 research group answering this question (Table 1 ). So improvements are needed for the $\mathrm{L} 2$ research group.

Regarding patient follow-up, statistical data show that both research groups need to be improved, more obviously group L2, which interprets the radiological differences compared to previous examinations only as $59.8 \%$ versus $88.5 \%$ for group L1 research (Table 1). Follow-up care in the radiology report is extremely important, because the tactics of subsequent treatment to which the patient will be subjected depend on this aspect. A qualitative follow-up demonstrates the role of the radiologist, but also enhances the interdepartmental relationship in the management of the pathology.

Table 1 The difference between the specific oncology data in the radiological reports of L1 and L2 groups.

\begin{tabular}{|l|l|l|l|}
\hline $\begin{array}{l}\text { Specific oncological data in the } \\
\text { radiology report }\end{array}$ & L1 group & L2 group & Comments \\
\hline $\begin{array}{l}\text { Specification of scanning protocols in the } \\
\text { radiology report }\end{array}$ & $0 \%$ & $95,3 \%$ & Requires implementation for L1 group \\
\hline $\begin{array}{l}\text { Specifying the cause of the examination } \\
\text { in the radiology report }\end{array}$ & $100 \%$ & $26 \%$ & Needs improvement for L2 group \\
\hline $\begin{array}{l}\text { Use of international terminology in the } \\
\text { radiology report }\end{array}$ & $0 \%$ & $74 \%$ & Requires implementation for L1 group \\
\hline \begin{tabular}{l} 
Oncological follow-up \\
\hline
\end{tabular} & $88,5 \%$ & $59,8 \%$ & $\begin{array}{l}\text { Needs improvement for both groups, } \\
\text { more obviously for L2 }\end{array}$ \\
\hline
\end{tabular}

\subsection{SWOT analysis of the radiology reports}

After interpreting the collected statistical data we have identified several non-conformities, but also very well-marked elements in the examined radiology reports, so we have decided to systematize the information through a SWOT analysis (Table 2), which originates from a research conducted between 1960 and 1970 at the Stanford Research Institute in the USA. The acronym SWOT is derived from "Strengths, Weaknesses, Opportunities, Threats". SWOT analysis also has its limitations, because categorizing certain aspects as strengths, weaknesses, opportunities and threats could be very subjective due to a high degree of uncertainty. Following the SWOT analysis of the radiology reports, we have developed a standardized model for describing CT images of patients with oncological pathologies of the abdomen and pelvis. The decision-making process was built on Strengths, eliminating Weaknesses, exploiting Opportunities and removing Threats. 
Table 2 SWOT analysis of radiological reports of patients with oncological pathologies of the abdomen and pelvis.

\begin{tabular}{|c|c|}
\hline $\begin{array}{l}\text { Strengths } \\
\text { - Access to advanced technologies, such as } \\
\text { high-performance CT equipment, which } \\
\text { allow detailed viewing of the studied } \\
\text { structures; } \\
\text { - Integrated PACS and RIS PACS information } \\
\text { system, and modern post-processing } \\
\text { programs; } \\
\text { DLP is indicated in the radiological report } \\
\text { for the possibility of calculating the } \\
\text { cumulative dose. }\end{array}$ & $\begin{array}{l}\text { Weaknesses } \\
\text { - Lack of an action guide in the radiological } \\
\text { examination of cancer patients; } \\
\text { - Lack of a standardized oncological scanning } \\
\text { algorithm; } \\
\text { - Not all radiological reports indicate the patient's } \\
\text { medical history, indications for CT examination } \\
\text { - Selective interpretation of follow-up; } \\
\text { Therapy response is not specified according to } \\
\text { international terminology; } \\
\text { The conclusions are a summary of the medical } \\
\text { report. }\end{array}$ \\
\hline $\begin{array}{l}\text { Opportunities } \\
\text { - Implementation of the "oncological } \\
\text { assessment" as a result of the study; } \\
\text { - Growing demand for abdominal+pelvic CT } \\
\text { examinations as a result of the study } \\
\text { outcomes; } \\
\text { - Access to technological innovation Mint } \\
\text { Medical, artificial intelligence software that } \\
\text { contributes to consolidation and } \\
\text { specialization in oncology. }\end{array}$ & $\begin{array}{l}\text { Threats } \\
\text { - Lack of a National Cancer Register to compare the } \\
\text { authenticity of specific oncological data; } \\
\text { - Incomplete access to the oncological history of the } \\
\text { examined patients (medical records, CD holder of } \\
\text { previous examinations, postoperative extracts) } \\
\text { secondary to their conscious or unconscious non- } \\
\text { cooperation; } \\
\text { - Limited experience of medical staff with } \\
\text { oncological pathology. }\end{array}$ \\
\hline
\end{tabular}

\subsection{Improved radiology report - standardized model}

Consequently, standardized radiology reports of patients with oncological pathologies of the abdomen and pelvis examined by computed tomography should contain the following mandatory fields:

- Passport data (name and surname, age, date of examination); received radiation dose data such as DLP; the examined region (abdomen or abdomen + pelvis); use of contrast agent (type, dose, method of administration); indications for examination; history of the disease (date, year, name of surgery, specific post-intervention therapies).

- Description of each organ in the scan field: liver (positioning, right/left lobe size, contour, structure, parenchyma density in pre- and postcontrast sequences); characteristic of intrahepatic bile ducts; gallbladder (size, contour, structure, wall thickness); diameter of the portal vein, abdominal aorta, inferior vena cava and the splenic vein; pancreas (size of the head, body, tail, contour, structure, characteristics of the pancreatic duct); spleen (size with the calculation of the splenic index, contour, structure); adrenal glands (shape, size); kidneys (size, location of each kidney, structure of the pelvicalyceal system).

- Evaluation of intra-abdominal lymph nodes, bone changes in the thoraco-lumbar segment of the spine in the scanning field are reported. The presence or absence of free intraperitoneal fluid is determined. The imaging sections of the lungs and soft tissues in the scanning field, with the respective specifications, must be followed.

- For women - specify how many days the menstrual cycle lasts. Describe each organ separately, the uterus (position, size, contour, diameter of the cervical canal); ovaries (contour, size); the characteristic of the Douglas space with the specification of the presence/absence of the liquid in the projection; bladder characteristics (contour, structure, wall dimensions).

- For men - characteristics of the prostate (size, contour, structure, signs of capsular extension); seminal vesicles (size, shape, structure); bladder (contour, structure, wall dimensions).

- The condition of the perirectal adipose tissue is also evaluated in the ischiorectal fossa as well as the regional lymph nodes. Bone changes are reported in the lumbosacral segment of the spine.

- If a tumor is identified in the scanning field, it is characterized in detail as follows - the exact topographic location, indicating all adjacent structures in the three anatomical planes, specifying the 
compression/infiltration of adjacent structures; the presence/absence of perilesional edema and its side effects; three-dimensional diameters (transverse, anteroposterior and craniocaudal); shape, contour, structure.

- In examinations that use contrast material, the post-contrast sequences are characterized with the detailed description of the structural, dimensional, shape changes of the described tumor. For multiple lesions, indicate their exact number at the time of scanning.

- In conclusion, the pathologies described in the text of the radiology report should be listed, with the recommendation to be correlated with the clinical, anamnestic and laboratory data and the consultation of the specialist doctor for a complete, complex diagnosis and its subsequent oncological management.

- Compare the current CT images with the previous CT images, specifying the treatment response, if appropriate, in accordance with the international terminology.

- At the end - the signature and initials of the radiologist.

\section{Conclusion}

The abdominal and pelvic CT examination with contrast is the investigation of choice for tumor identification, local metastases, distant spread [6], staging and follow-up of these patients. Thus, the qualitative interpretation of CT images with detailed delimitation of the primary tumor, in order to determine its resection capacity, and assessing the presence of metastatic spread that would change the surgical approach, or the mandate of non-surgical treatment - are the direct tasks of the radiologist. This information defines applicable therapeutic strategies and provides a guide to the patient's prognosis.

\section{Compliance with ethical standards}

\section{Acknowledgments}

I would like to express my deep gratitude to Professor Davide Caramella, my research supervisor, for his patient guidance, enthusiastic encouragement and useful critiques of this research work. I would also like to thank Dr. Giulia D’Agostino and Dr. Andrei Scripnic for their help in data collection.

\section{Disclosure of conflict of interest}

There is no conflict of interest.

\section{Statement of informed consent}

Informed consent was obtained from all individual participants included in the study.

\section{References}

[1] McLaughlin CP and Kaluzny AD. (2006). Continuous Quality Improvement in Health Care: Theory, Implementations and Applications, 3rd edition. Sudbury, MA: Jones and Bartlett Publishers Inc, 3-40.

[2] Mosadeghrad AM. (2012). A conceptual framework for quality of care. Mater Sociomed, 24(4), $251-61$.

[3] Mosadeghrad AM. (2012).Towards a theory of quality management: an integration of strategic management, quality management and project management. International Journal of Modelling in Operations Management, 2, 89-118.

[4] Donabedian A. (1980). The definition of quality and approaches to its assessment. Volume 1. Ann Arbor: Michigan Health Administration Press.

[5] Donabedian A. (1988). The quality of care. How can it be assessed? JAMA, 260(12), 1743-8.

[6] Rodney JH, Robert EW and Eddie WFL. (2006). PET/CT: will it change the way that we use CT in cancer imaging? Cancer Imaging, S52-62. 